

\title{
REPRESENTACIONES SOCIALES DE LA INCLUSIÓN ESCOLAR DE ESTUDIANTES CON LIMITACIÓN VISUAL: CREENCIAS Y PRÁCTICAS
}

\section{Social Representations of School Inclusion Students with Vision Impairment: Beliefs and Practices}

Fecha de Recepción: 19 de Noviembre de 2011 - Fecha de Aprobación: 8 de Marzo de 2012

\begin{abstract}
RESUMEN
Esta investigación tuvo como objetivo comprender las Representaciones Sociales que tienen los diferentes actores de la comunidad educativa de 10 colegios públicos de Bogotá, frente a las experiencias de Inclusión Escolar de estudiantes con limitación visual. Las representaciones sociales son definidas como maneras específicas de entender y comunicar la realidad. Se realizo un estudio de enfoque cualitativo, hermenéutico- interpretativo; la estrategia de Investigación fue el estudio de caso y la técnica de recolección de información fue la entrevista de grupo focal. Se realizó un Análisis de Dominio (Spradley, 1979) que permitió comprender las representaciones sociales presentes en el discurso de los participantes. La información obtenida fue analizada a partir de las siguientes categorías: Necesidades y Expectativas, Prácticas y Significado de la discapacidad. Los resultados de esta investigación permiten concluir que si bien, existen contenidos compartidos en algunas de las representaciones sociales que han construido los diferentes actores de la comunidad educativa en relación con los procesos de inclusión escolar de estudiantes con limitación visual, también evidencian diferencias entre ellos a partir de su rol, experiencia y relación con esta condición. Se generó conocimiento en relación con las creencias y prácticas educativas que tienen los diferentes actores involucrados en las experiencias de inclusión escolar de personas con limitación visual.
\end{abstract}

PALABRAS CLAVES

Representaciones Sociales, Inclusión Escolar, Discapacidad Visual

\begin{abstract}
The aim of this research study was to understand the social representations of different stakeholders at 10 Bogota's public schools, related with students' experiences all of them with visual impairment. Nowadays, Social representations are defined as specific ways to understand and communicate the reality. We realized a qualitative study approach which research strategy was the case study and data collection technique was the focus group interview. We used adomainanalysis(Spradley, 1979)by understand the social representationsin participant's discourse. The information obtained wasanalyzed fromthe following categories: Needs and Expectations, Practices andMeaningof Disability. By our results we concluded that in spitethere aresomeshared contentin social representations there are somedifferences between different stakeholders associated with theirrole, experience and relationship with this condition. New knowledge was generated about educational practices and beliefs related with differents takeholders involved in the experiences of educational inclusion of students with visual impairment.
\end{abstract}

KEY WORDS

Social Representations School Inclusion, Visual Impairment 
S in duda el reto de las sociedades actuales es hacer realidad el derecho a la educación de todos los niños y niñas, se espera que la oferta educativa, de respuesta a la individualidad y evite la discriminación por diferencias de género, nacionalidad, religión, o condiciones de discapacidad de origen cognoscitivo, físico o sensorial.

Desde ese planteamiento, Colombia se comprometió con una política educativa incluyente, legitimada desde los derechos del niño y desde la Ley general de educación. (1994). Sin embargo, si bien es cierto que el marco legal es un punto de partida para hacer visible el derecho que tiene la población con discapacidad a la educación, el hacer realidad el ejercicio de éste, supone la generación de unas condiciones institucionales que garanticen su cumplimiento.

A pesar de este reconocimiento, el compromiso asumido en Colombia para la integración y la inclusión educativa ha dado resultado, el país cuenta hoy con un CONPES para discapacidad y con varios planes de acción estratégicos, por ejemplo el Plan estratégico 2011 - 2014: "Inclusión educativa y sociocultural de la población con discapacidad visual"- INCI, vale la pena, resaltar que hay aun falencias, vacíos y espacios que requieren ser abordados desde la investigación, para poder generar conocimiento y plantear propuestas en la resolución de estos. Dentro de los logros obtenidos cabe destacar el trabajo del Ministerio de Educación, las Secretarías de Educación e instituciones como el INCI, vía a la consolidación del derecho a la educación de la población con discapacidad visual.

Algunas cifras revelan los avances, según el Sistema de Información de Matrículas del Ministerio de Educación (SIMAT) para el año 2010, 11.556 niños con discapacidad visual obtuvieron ingreso al sistema público educativo (1.047 ciegos y 10.509 con baja visión) y en 2011 el programa PAIPI de atención a la Primera Infancia, prestó servicios de atención a 82 niños con discapacidad visual en 17 departamentos del País, cifra muy baja en relación con datos del DANE que señalan que más de 3469 niños y niñas deberían estar matriculados en el Prejardín. (Buitrago, 2011).

El Instituto Nacional para Ciegos -INCI- señala que pese a estos avances, existen dificultades muy arraigadas en relación con aspectos como la cobertura, altas tasas de deserción (refieren una tasa del $45,5 \%$ para el periodo comprendido entre 2006 y 2009) y la calidad en los procesos educativos, señalan la fuerte necesidad de generar estrategias de mejoramiento, garantías para el cumplimiento pleno del derecho a la educación, reestructuración en los colegios, tanto a nivel físico como de personal, desarrollo de competencias, enfoque diferencial y de integralidad, eliminación de las barreras de acceso y de los procesos de exclusión y transformación en los imaginarios sociales (Buitrago, 2011).

La humanidad a lo largo de su historia ha construido diferentes Representaciones frente a las personas con discapacidad, permeadas por las explicaciones que sobre ella se han elaborado. Esta población ha sido vista como sujeto de compasión, de caridad, producto de un castigo o de inspiración divina. Cada una de estas miradas ha determinado una forma de relación, es así como practicas tales como el encierro, el rechazo, el miedo y la discriminación han existido dependiendo del contexto social, político y cultural del momento histórico y han significado para esta población la exclusión de espacios, que como la escuela regular, permiten la participación de las personas en las dinámicas de la comunidad a la que pertenecen (Quinn y Degener, 2002) Solamente es desde la década de los 70, cuando en algunos países de Europa y Estados Unidos, se empieza a promover el acceso de personas con discapacidad al sistema educativo regular (Woolfolk, 1999; Shea y Bauer, 1999). En los años 9o La Conferencia Mundial sobre la Educación para todos, en el artículo 1 y 3 reivindica el derecho que tienen todas las personas al aprendizaje (herramientas y contenido) de tal manera que puedan desarrollarse plenamente en la sociedad a la que pertenecen; además explicita la necesidad de generar condiciones de igualdad que garanticen que las personas con discapacidad tengan acceso al sistema educativo(Declaración mundial sobre educación para todos, 1990).

Sin duda, el paradigma frente a las personas con discapacidad se ha ido transformado poco a poco; de una mirada centrada primordialmente en la rehabilitación física, a una mirada en la que se contemplan otros aspectos, como son las barreras culturales y sociales a las que tienen que enfrentarse estas personas, y que inciden significativamente en sus posibilidades reales de participación (Verdugo, 2003). (Vaca, Còrdoba, Rosero, Gòmez y Lucas, 2011; Moreno, 2007).Por esta razón el acercarse a comprender las Representaciones Sociales que circulan y se construyen en espacios escolares en los que participan personas con discapacidad, grupo especialmente vulnerable a la violación de los derechos, (en este caso el derecho a la educación), permitirá generar propuestas de intervención dirigidas a resignificar aquellas que reproducen formas de interacción excluyentes en este contexto..

Las Representaciones Sociales como sistemas cognitivos con una lógica y un lenguaje propio, dan cuenta, como lo señala Jodelet (1987) de "la manera como nosotros sujetos sociales aprehendemos la vida diaria, las características de nuestro ambiente, las informaciones que en el circulan e identificamos a las personas de nuestro entorno próximo o lejano" ( $\mathrm{p} 474)$. Son definidas, como maneras específicas de comprender la realidad, un saber del sentido común que se orienta hacia la comunicación y el dominio del entorno (Jodelet, 1987). En este sentido Moscovici (1987), afirma que las representaciones sociales cumplen una doble función, por un lado establecen un orden que le permite al individuo orientarse en el mundo social y dominarlo, y de otro posibilitan la comunicación entre los individuos de una colectividad. Este mismo autor considera que en el interior de los grupos, existe un conjunto organizado de cogniciones relativas a un objeto o a una realidad, cogniciones que son compartidas por sus miembros; no son solo productos mentales, sino que pueden ser entendidas como construcciones simbólicas que se crean y recrean en el curso de las interacciones sociales.

Las representaciones sociales, se construyen a partir de un conjunto de valores atribuido a una imagen, el cual se organiza formando una estructura conceptual que da forma a las normas y reglas que orientan el obrar de los integrantes de una comunidad y que además les garantiza su permanencia y aceptación en la misma. Es así como, Moscovici (1987) reconoce la presencia de dos componentes en la representación Social, el primero de ellos hace referencia a su 
contenido (informaciones y actitudes) y el segundo se relaciona con la organización de este contenido, es decir su estructura interna.

El contenido y la estructura de la representaciones sociales se conforma a partir de referentes como las condiciones y los contextos en las que aparecen, las experiencias, las informaciones, las tradiciones, es decir de la red de significados que circulan en la cultura; se constituye de esta manera en un conocimiento socialmente elaborado y compartido, que guía las interacciones; pueden considerarse como la versión contemporánea del sentido común (Moscovici, 1987).

Las representaciones sociales que un conjunto de individuos posee acerca de determinado objeto, se encuentran en un continuo flujo dialéctico con la práctica de ciertas costumbres y conductas alrededor de estas cogniciones compartidas (Vaca, Chaparro y Pérez, 2006). Es esta relación dialéctica entre interpretaciones y actos, entre individuos y contextos sociales, la que hace que el estudio de las representaciones sociales sea de utilidad en la comprensión de problemáticas comunitarias, como lo es la de la discapacidad, pues permite comprender cogniciones y sistemas explicativos que median las interacciones en determinados grupos.

En Colombia, ha habido una serie de problemas que han afectado los procesos de inclusión escolar y con esto, la inclusión de los niños y las niñas con discapacidad visual en las aulas regulares de clase. El primero de ellos ha sido los escasos e insuficientes desarrollos de políticas públicas para la discapacidad; el segundo problema ha sido la poca transformación de la sociedad colombiana y sus representaciones sociales en torno a la discapacidad, la prevalencia una cultura excluyente basada en imaginarios, actitudes y prácticas que no favorecen la inclusión y que requiere una urgente intervención; un tercer problema ha sido los bajos niveles de participación y empoderamiento de la población con discapacidad visual en los procesos políticos, educativos, sociales y económicos (Buitrago, 2011)

En este marco de referencia se considera que las representaciones sociales de la comunidad educativa acerca de la inclusión escolar de personas con discapacidad y en este caso de discapacidad visual, afectan la manera como se constituyen las prácticas escolares y por tanto pueden favorecer los procesos de inclusión de la escuela o por el contrario perpetuar prácticas segregadoras en su interior.

La defensoría del Pueblo (2004), realizó un estudio para evaluar los procesos de integración educativa de niños y niñas con discapacidad en Bogotá, desde la perspectiva de los derechos. Las conclusiones de este estudio señalan que la aplicación de las políticas de integración en Bogotá, "no son plenamente coherentes con los principios de no discriminación, trato preferente y adopción de medidas positivas para asegurar el respeto, la protección y la realización progresiva del derecho a la educación de los niños y niñas con discapacidad" (p. 89).Los resultados muestran además, que existen déficits en relación con los componentes fundamentales de la inclusión educativa: disponibilidad, accesibilidad, aceptabilidad y adaptabilidad, en razón de que los establecimientos educativos "integradores" no poseen maestros especializados de apoyo y hay ausencia de soportes pedagógicos acordes a las necesidades educativas de los estudiantes con discapacidad. En cuanto al acceso se reporta la existencia de restricciones en el proceso de matrícula de los niños y niñas con discapacidad, por motivos como la falta de cupos o falta de experiencia en procesos de integración escolar.
También se evidenció en el estudio que existen barreras de tipo social, como dificulta para acceder a actividades escolares, particularmente deportivas y/o culturales, así como actitudes discriminatorias hacia las personas con discapacidad por parte de los miembros de la comunidad educativa.

Por su parte, Vallejo (2005), identificó tres aspectos centrales de las representaciones sociales que los docentes de educación básica construyen acerca de la discapacidad, sintetizándolos en los siguientes aspectos: a) el discapacitado es otro distinto al nosotros, b) La identificación de la diferencia en la carencia o la disfunción y c) la diversidad en el sentido de variación. Estos componentes de las representaciones se traducen en acciones de exclusión de las personas con discapacidad, particularmente en los espacios escolares en donde se espera encontrar espacios que promuevan la valoración aceptación y reconocimiento de las diferencias dentro de un marco de equidad para todos los niños y las niñas.

En está misma línea existen algunas investigaciones que señalan la importancia de la integración escolar para la inclusión social de las personas con discapacidad, por ejemplo, García y García (1991), encontraron que el tipo de actividades escolares está relacionado con situaciones de rechazo o aceptación de estudiantes con discapacidades por parte de sus compañeros de clase. Es así como en situaciones típicas de estudio o de trabajo académico, las personas con limitación son menos rechazadas que en situaciones de juego, sin embargo Checa y Ato (1995), realizaron un estudio por medio de sociogramas, en el que reportaron que los niños ciegos son menos elegidos para tareas de tipo académico que los compañeros videntes, a pesar de no existir un rechazo explícito hacia ellos.

Delgado (2007), realizó un estudio que tuvo como objetivo explorar las representaciones sociales acerca de la ceguera en seis grupos poblacionales diferentes, a saber: personas ciegas, personas del sector productivo que tienen a su cargo funciones de selección y contratación de personal, familias de personas ciegas, funcionarios del sector salud, educación especial y bienestar social, estudiantes de colegios públicos a través de entrevistas en profundidad y Grupos Focales. También se realizó una encuesta a 225 personas para indagar sobre percepciones, creencias, opiniones y expectativas de ciudadanos comunes.

Los resultados muestran que la autopercepción de las personas ciegas se construye a partir de variables como el nivel social, las experiencias de socialización primaria y secundaria y las experiencias previas de inclusión y exclusión. En el sector empresarial, se encontró desconocimiento de lo que es la ceguera y de sus posibilidades de inclusión en el mundo laboral, las representaciones están enmarcadas dentro de un estereotipo de Limitación/superación, que se fundamenta positivamente o negativamente.

Por otra parte, las representaciones que construyen las personas que no han tenido contacto con personas ciegas están centradas fundamentalmente en estereotipos (lástima, incapacidad, dependencia) que llevan a prácticas sociales excluyentes. Mientras que las representaciones de los funcionarios que trabajan con población ciega están permeadas por el discurso técnico - profesional, en el que el contenido de la representación hace referencia a aspectos de tipo médico, o en otros casos se relaciona con aspectos sociales. 
Este estudio evidencia la importancia de la inclusión escolar, como experiencia de socialización, en la autopercepción que construyen las personas ciegas y su relación con la inclusión social, resultado relevante para el objetivo de esta investigación.

Estas investigaciones reflejan que el derecho a ser incluido en la escuela regular no garantiza la plena inclusión social del sujeto en el ámbito educativo, Existen creencias y significados arraigados en la cultura que requieren ser develados, comprendidos y deconstruidos, para orientar acciones que promuevan el cambio en prácticas escolares que permitan pasar de una escuela integradora a una escuela inclusiva.

En concordancia con lo expuesto se planteó la siguiente pregunta de Investigación: ¿Cuáles son las Representaciones Sociales que tienen los diferentes actores de la comunidad educativa (padres, maestros, docentes de apoyo y alumnos con limitación visual y sus pares) frente a las experiencias de Integración Escolar de niños con limitación visual?

El objetivo de la investigación fue comprender las Representaciones Sociales de la comunidad educativa de padres maestros y alumnos de 10 Colegios del Distrito, en relación con al experiencia de inclusión escolar de estudiantes con limitación Visual, con el propósito de generar, con la comunidad una propuesta educativa orientada a favorecer los procesos de Inclusión en dichos espacios escolares.

\section{METODOLOGÍA}

\section{Tipo de Investigación}

Se utilizó un método de carácter cualitativo, con enfoque hermenéutico-interpretativo. Este tipo de investigación se interesa por acercarse a la realidad que está siendo estudiada a través de de la aprehensión que hacen los participantes de ella. (Ashworth, 2008). Estas interpretaciones se conceptualizan como proposiciones lingüísticas que dan cuenta de la manera como las personas construyen la realidad.

\section{Participantes}

Padres, maestros y alumnos de educación media de 10 colegios del distrito capital que participan en experiencias de inclusión escolar de estudiantes con limitación visual. La selección de los participantes fue intencional y se realizó partiendo de la clasificación propuesta por Valles (1997), en la que define tres tipos generales de entrevistados: entrevistados claves, entrevistados especiales y entrevistados representativos. Se utilizó el criterio de "entrevistado especial", definido como cualquier persona que da información relevante para los objetivos del estudio y es seleccionada porque ocupa una posición única en la comunidad. (Valles ,1997).

Técnicas de Recolección de Información

Entrevista de grupo focal

La entrevista de Grupo Focal se define como un encuentro presencial de un grupo de personas, que discuten en relación a un tema
(Wilkinson, (2008). Valles (1997), señala que este instrumento de investigación es utilizado cuando los entrevistados han sido expuestos a una situación social concreta, no controlada pero observada.

Se orienta a comprender las percepciones, significados y prácticas que tiene las personas frente a las experiencias de Inclusión Escolar. Para la realización de las sesiones de Grupo Focal, se elaboró una guía de preguntas estructurales (Bogan y Taylor, 1994). Esta guía fue flexible y se orientó a recoger el flujo de información particular de cada grupo de entrevistados (Valles, 1997).

Procedimiento

La Investigación se llevó a cabo en las siguientes fases:

1. Fase 1: Selección de la muestra: la selección de la muestra fue intencional teniendo en cuenta los objetivos de la investigación.

2. Fase 2: Realización de los grupos Focales, trascripción y análisis de las narrativa:Una vez obtenido el consentimiento informado por parte de cada uno de los participantes, se realizaron 27 Grupos Focales,,en diez instituciones educativas del Distrito que participaron en la Investigación. Cada uno de estos grupos estuvo formado por los diferentes actores participes en la experiencia de Inclusión Escolar: Se realizaron 6 grupos focales a los Padres, 6 a los Docentes, 7 a los compañeros de los estudiantes con limitación visual, 7 a los estudiantes con limitación visual y 1 a las profesoras de Apoyo (Tiflólogas).

3. Análisis de la información recolectada en los grupos focales: La narrativa obtenida en los grupos Focales, fue analizada a partir del Análisis de Dominios, siendo éste una clase análisis etnográfico, que se propone descubrir sistemas de significados culturales que se encuentran inmersos en un grupo. Involucra una búsqueda exhaustiva de las unidades de conocimiento cultural definidas como dominios (Spradley, 1979). Este análisis permitió situar los ejes o factores generales que bajo los cuales se encontraba organizada la representación social. Esta fase correspondió al punto de vista émico de las experiencias de los participantes, un acercamiento desde su referencia, el cual se complementó con el punto de vista de los investigadores como agentes externos, que corresponde a la perspectiva ética. Se respetó el anonimato de los participantes.

\section{RESULTADOS}

Los resultados obtenidos se analizaron a partir de las siguientes categorías: Necesidades y Expectativas, Prácticas Relacionales y Significado de la discapacidad. Al interior de cada una de estas categorías, emergieron los dominios que como unidades de conocimiento cultural organizan la representación que tienen los participantes de estos aspectos.

\section{Necesidades y Expectativas}

Esta categoría da cuenta de las necesidades que tiene la comunidad educativa para hacer realidad los procesos de inclusión escolar de los estudiantes con discapacidad visual.

El análisis de la narrativa de los participantes, permitió establecer la existencia de una serie de necesidades que corresponden a aspectos 
como capacitación, recursos tanto humanos como pedagógicos e infraestructura. De ésta categoría dan cuenta los siguientes dominios: "Defiéndase como pueda" y "Nos deberían preparar".

\section{Dominio: "Defiéndase como pueda"}

En relación con la capacitación las narrativas encontradas en los Grupos Focales realizados a los diferentes actores de la comunidad educativa, coinciden en señalar la necesidad de capacitación especialmente identificada para la comunidad de docentes ( 6 citas); las siguientes afirmaciones de dos de estos participantes ilustran este aspecto:

“...necesitamos estrategias, porque aquí vienen a hablarnos maravillas y análisis de lo que son los niños estos, pero para el trabajo en el aula no tenemos casi nada, necesitamos que nos digan directamente que hacemos con ellos, pero que nos capaciten de verdad; que nos enseñen a preparar materiales" y ".....yo pienso que lo primero que se debe tener en cuenta es una capacitación, por ende a nosotros, empezando porque aquí se tuviera el grupo de apoyo, que eso muchas veces se habló, yo por ejemplo decía listo ustedes reciben los niños con necesidades especiales, pero lo mínimo que deberían hacer era capacitar al maestro y decirle mire, el niño que va para su salón estas son las necesidades, estas son las dificultades que él tiene, a uno simplemente le decían tome y defiéndase usted como pueda....".

En este mismo sentido se encuentran narrativas de los participantes del Grupo Focal realizado con los compañeros (4 citas) y del grupo focal realizado con los padres de familia (6 citas). Al respecto uno de los compañeros afirmó:

“....preparar a los profesores, porque ellos no saben si les llega un niño con limitación visual, entonces prepararlos para saber que los niños se sientan más cómodos" y "...lo principal sería que todos los profesores aprendieran el método, eso sería lo principal" afirmo una de las madres participantes.

En la narrativa de los estudiantes con limitación visual, se encuentran también afirmaciones referidas a la necesidad de capacitación de los profesores (2 citas) uno de ellos expresó:

\section{"...los profesores no tienen los recursos para explicarle a uno, aunque quieran explicarle a uno no saben cómo"}

Por último las profesoras de apoyo identifican la necesidad de capacitación en la implementación de nuevos modelos pedagógicos ( 5 citas), que den lugar a prácticas diferentes a las tradicionales y metodologías que den respuesta a las preguntas de los profesores. Las siguientes citas ilustran esta apreciación:

“...estamos en unos procesos pedagógicos en la escuela todavía, con un modelo muy tradicional" ; y "... seguimos haciendo la misma práctica. hay una cantidad de métodos, y surgen muchas preguntas para la que el que el maestro no las tiene."

Dominio: nos deberían preparar

Se identifican, otras necesidades particularmente en la narrativa de los compañeros (6 citas) relacionadas con la necesidades de capacitación para ellos, orientada a proporcionarles información sobre la forma como deben relacionarse con sus compañeros con limitación visual. Las siguientes frases expresadas por los compañeros participantes dan cuenta de éste hallazgo:

"me parece que a nosotros también nos deberían preparar como para saberles hablar a ellos, saberlos como tratar, porque no todas las personas son como nosotros, no todas las personas los van a tratar bien, entonces como que nos preparen...; y" porque digamos yo le puedo decir a él, ¿tu porque no ves? Entonces pues yo me sentiría mal entonces nosotros tampoco es que sepamos mucho sobre el tema, no estamos bien informados sobre el tema".

También los padres identifican la necesidad de capacitación para ellos, específicamente en relación con los procesos de comunicación y asesoría especializada dirigida a facilitar las interacciones entre los miembros de la familia que tiene un hijo con limitación visual ( 5 citas), al respecto uno de ellos señaló:

“...se podría trabajar no solo con los niños sino con el núcleo, no solo con la mama sino con el núcleo por ejemplo papá, mamá, hermanos porque yo tenía mucho problemas con mi hijo porque le decía a mi hija "Mongólica" por lo de su problemita del aprendizaje y yo sufría mucho, y entonces por eso ella se echaba para atrás entonces yo sugeriría trabajar más con el núcleo".

Otro de los aspectos señalado por los participantes en el que se identifican necesidades es el relacionado con los recursos tanto humanos como pedagógicos. Se encuentran diferencias en las necesidades identificadas por cada uno de los grupos de los actores participantes. Es así como los docentes señalan la necesidad de contar con el apoyo de un equipo interdisciplinario que de soporte a los procesos de inclusión (9 citas), pues consideran que el trabajo que realizan las profesoras de apoyo es insuficiente (6 citas). Estas necesidades se ilustran en las siguientes afirmaciones realizadas por algunos de los docentes participantes:

“... pues yo pienso que el rol primordial del grupo de apoyo es ante todo la capacitación hacia los docentes, yo pienso que ese es el rol primordial, y dentro de esa capacitación, el acompañamiento, que sea un acompañamiento continuo, digamos en el aula de clase"; "el trabajo de ella (refiriéndose a la tiflóloga) es excelente; "es insuficiente a nivel de personal y se necesita porque tenemos bastantes niños" y "Es importantísimo que haya alguien que le ayude a uno, pues si, esta la tiflóloga, pero como le digo ella con tantos niños no puede, por ejemplo allá en la sede de la frontera ahí un niño cieguito yo no le dicto clase. No debería haber una persona sino un grupo de apoyo".

En relación con la existencia y utilización de recursos pedagógicos tanto los estudiantes con limitación visual (4 citas) como los profesores ( 2 citas) señalan en la narrativa de los grupos focales, la insuficiencia de materiales tanto didácticos como tecnológicos para responder a las necesidades de los estudiantes con limitación visual. Las siguientes afirmaciones dan cuenta de este reconocimiento por parte de los estudiantes con limitación visual: 
“...en sociales a mí se me dificulta mucho, pues se necesitan libros y yo no puedo leer, falta más dotación de libros y "... lo que no me gusta es que no hay una maquina Terry"

En ese mismo sentido uno de los profesores afirmó:

"La problemática ya es más con los aspectos de elementos de trabajo, las guías que decía la profesora, eehh los elementos de audiovisuales, de computo...es muy difícil trabajar eso con ellos."

También los docentes participantes perciben una sobrecarga laboral, producto de las exigencias que les demanda el tener en el aula estudiantes con limitación visual (3 citas), exigencias para las cuales, como se mencionó anteriormente, consideran que no están preparados. Las siguiente afirmación da cuenta de esta apreciaciones:

"...entonces a la hora de la verdad a nosotros nos toca hacer un montón de cosas, por las cuales ni tenemos tiempo ni estamos capacitados para eso".

En este mismo sentido se encontraron narrativas ( 5 citas) en los grupos focales realizados con los padres, quienes consideraron que la carga de trabajo de los docentes no les permitía atender adecuadamente a los estudiantes con limitación visual. Afirmaciones como:

"Hay profesores que manejan 40 estudiantes y varios cursos, la carga es muy grande y esto impide que puedan dedicar más tiempo a los niños, deberían contar con más apoyo".

En relación con las necesidades de infraestructura identificadas en la narrativa de los grupos focales se encontraron los siguientes resultados: los estudiantes con limitación visual reportan la existencia de muros especialmente en el patio de recreo, que obstaculizan su movilidad (4 citas) las siguientes dos citas dan cuenta de esa afirmación:

"Yo cambiaría ese muro que está allá, con el que me casqué que día (Risas)....Eso ni con bastón ni con nada se puede" y "me gustaría que hubiera otro patio para movilizarnos mejor, porque digamos sí, uno va caminando lo mas de normal en el patio y toda la gente quiere empujarlo a uno".

También se encuentran narrativas en los grupos focales realizados con los compañeros, que hacen referencia a la necesidad de adecuar los espacios físicos para sus pares invidentes (2 citas) y de facilitar su movilidad de la población con limitación visual a través de la utilización de sonidos (2 citas). Las siguientes citas ilustran éste aspecto:

"Estas instalaciones no están tan preparadas, porque en cualquier momento digamos, un día de estos un niño llega a bajar por las escaleras se cae y qué. ¿Quién responde? Entonces también deben tener una seguridad como más...que les den esa confianza de que ellos pueden desenvolverse más en otro espacio" y "...yo creo que deberían poner baños para invidentes con sonidos para que ellos sepan si están sucios o no, poner un baño que solo sea para invidentes, para que otros no entre, porque se entran ahí, poner en los salones".
Prácticas Incluyentes vs Prácticas Excluyentes.

Esta categoría da cuenta de las interacciones cotidianas que tienen los diferentes actores de la comunidad educativa, con relación a los procesos de aprendizaje e integración social y familiar del estudiante con limitación visual. También da cuenta de las acciones de la institución educativa y las entidades distritales y de las políticas gubernamentales que buscan la inclusión de los estudiantes con limitación Visual. Fueron analizadas tres tipos de Prácticas: prácticas relacionales, practicas pedagógicas y practicas Institucionales. El análisis de la narrativa permite concluir que en la vivencia de la cotidianidad al interior de las Instituciones educativas participantes, coexiste la presencia de prácticas incluyentes y excluyentes, que permean las interacciones de los diferentes actores de la comunidad educativa.

A continuación se presenta el análisis de la información obtenida en los grupos focales que corresponde a dos de los dominios pertenecientes a las practicas relacionales y a las prácticas pedagógicas que ocurren al interior de las Instituciones Educativas participantes.

\section{Practicas Relacionales}

\section{Dominio: Colaboración vs Indiferencia.}

El análisis de las narrativa por parte de los compañeros que participaron en los grupos focales, que da cuenta de las relaciones interpersonales que se establecen entre pares, muestra como algunas de ellas pueden ser consideradas como prácticas incluyentes pues buscan colaborar y apoyar el trabajo de los estudiantes con limitación visual (9 citas) sin embargo, como se mencionó anteriormente, éstas coexisten con otras prácticas que dan cuenta de la presencia de exclusión al interior de la Institución educativa (9 citas) traducida en comportamientos que buscan el evitar relacionarse con su compañero con limitación visual así, como la lectura negativa que hacen éstos, de los comportamientos del estudiante con limitación visual, por último la existencia de maltrato visible hacia el compañero con limitación visual. Las siguientes afirmaciones realizadas por los participantes del grupo focal realizado con los compañeros, dan cuenta de esta realidad:

"...en el caso de nosotros, con el grupito cuando estamos con él, tratamos de estar siempre con él, de estar siempre junto y de estar apoyando en lo que más se pueda y siempre compartir en los ratos de diversión, es lo que más hacemos, mas amigos que compañeros." y“... hay compañeras que se le sientan al lado y le dicen "ay mira, te dicto... mira..." le hablamos asî".

En ese mismo sentido algunos de los maestros destacan también la colaboración que se observa entre los niños hacia el compañero con limitación visual, así como los valores que fomenta la inclusión escolar (4 citas). Al respecto una de las profesoras se expresó de la siguiente manera:

"...ha generado en sus compañeros aceptación, ayuda, tolerancia, comunicación, hasta cuidado, porque ellos los cuidan, y algunos se responsabilizan de sus compañeros". 
El análisis de la narrativa, por parte de los estudiantes con limitación visual que participaron en los grupos focales, que da cuenta de las interacciones que establecen con sus compañeros, evidencia también la existencia de una tensión entre prácticas incluyentes ${ }_{5}$ Citas) y excluyentes (12). Es así como 5 de las citas encontradas dan cuenta de la colaboración y compañía que caracteriza algunas de sus relaciones, por ejemplo uno de ellos se expresó de la siguiente manera:

"...mi relación con los compañeros curso pues es muy chévere, porque ellos se preocupan por ayudarlo a uno, pues ellos también me tratan bien...osea si ellos necesitan algo pues me dicen y saben que también pueden contar conmigo, lo mismo yo con ellos"

Mientras que otros de los participantes se expresaron de la siguiente manera:

"Pues uno quiere salir con sus compañeros y pues es muy difícil salir con ellos al descanso, uno nunca sale con ellos al patio...sino que se queda aquí.", "Pero hay unas cosas que me disgusta Por ejemplo cuando estaba una compañera me dijo "Miguel échese para allá, y me hizo así y me pellizco..." y "...allá no le tienen a uno paciencia, era muy feo, me pegaban, me hacían bromas pesadas, como por ejemplo: echarme la maleta en la basura con todo y libros".

De la misma manera las narrativas encontradas en el grupo focal realizado con los padres de familia, reconocen que los compañeros se convierten en un soporte efectivo para el aprendizaje y adaptación de sus hijos con limitación visual (2 citas), pero consideran que éste soporte significa un costo para el compañero, al considerarlo una carga para él. La siguiente frase de uno de los padres participantes, ilustra esta afirmación:

"Pero igualmente un niño invidente se forma como una carga para los otros niños, los niños del salón de mi hija, ellos tienen que estar pendientes a la hora del descanso, ayudándola, entonces ahí es donde uno dice "si no fuera por esos niños, mi hija estaría sentada en una silla", sencillamente sentada en una silla, esperando que llegue la ruta"

Sin duda alguna, la inclusión escolar genera valores de solidaridad entre, compañeros, realidad que coexiste con otro tipo de prácticas que en contraposición, señalan como, los compañeros se pueden mostrar apáticos e indiferentes frente a sus pares con limitación visual, como se ilustra en las siguientes afirmaciones hechas por algunos de los compañeros y profesores que participaron en los grupos focales:

"Los aíslan cuando hay actividades en grupo" y "...porque lo evitan, como que cuando va hablar la gente prefiere que se vaya." "Cuando llegaron los niños de la otra sede, ellos son menos sensibles ante estas discapacidades, a ellos no les llama mucho la atención colaborar con estos niños con discapacidad, son un poco egoístas, son, no colaboran, ellos son muy, ¿Cómo decirlo? apáticos, indiferentes, hay mucha indiferencia y poca aceptación a estos niños".

La presencia de expresiones de maltrato (2 citas) fue reportada en las siguientes frases, la primera de ellas expresada por uno de los compañeros y la segunda por un estudiante con limitación visual: "...hay gente que...eehh va pasando un chico invidente y en vez de ayudarlo, le pasan por encima ó digamos...lo empujan o lo maltratan o le dicen groserías...cosas por el estilo" y "...no me gustan es como la hipocresía de algunos compañeros, como el trato, como el trato que le dan a uno, por decir que lo aceptan dentro de un grupo, pero a la vez como que le dicen: tranquilo, tranquilo que no hay que hacer nada, mientras que el docente ya había dictado la clase y había dictado las actividades, lo que tocaba hacer, lo "desescluyen" a uno, le dicen. No tranquilo fresco, lo tratan a uno como por esa hipocresía"

Los docentes además observan que los padres de los compañeros de los niños con limitación visual discriminan y excluyen a los estudiantes con limitación visual ( 2 citas), lo que evidencia la presencia de prejuicios en otros miembros de la comunidad educativa. $\mathrm{Al}$ respecto uno de los docentes se expresó de la siguiente manera:

"En los procesos de integración escolar es más difícil la aceptación por parte de los adultos que por parte de los niños, sobre las personas con necesidades educativas especiales, es más difícil que el padre de familia acepte el niño con necesidades educativa, que los mismos hijos, este es otro trabajo que el gobierno tienen que hacer con el adulto".

Prácticas Pedagógicas.

\section{Dominio: Compromiso vs desconocimiento}

El análisis de la narrativa de los grupos focales, en relación con las prácticas pedagógicas al interior de la Institución educativa da cuenta también de la existencia de diferentes realidades. Es así como algunos de los compañeros consideran que los profesores realizan ajustes en los procesos de enseñanza que favorecen la inclusión del estudiante con limitación visual. (4 citas), mientras que otros de los compañeros participantes piensan que los profesores no se comprometen con los alumnos con limitación visual: No les exigen o no realizan adaptaciones que favorezcan su aprendizaje (11 citas): las siguientes afirmaciones evidencian esta situación:

"....êl es como que "hay explíqueme" y muchos profesores le explican y le repiten, pero hay otros que "no, ahorita...en 10 minutos" y siempre lo dejan así y no les explican nada, entonces ese también es un gran problema. Hay profesores que le ayudan y otros que no. Hay profesores que les dicen en diez minutos y pasan como veinte" y "Los profesores como que intentan dejarle menos trabajos y el pasa sin ninguna complicación, si el no lleva ninguna tarea, pues normal no pasa nada"

Narrativas en este mismo sentido se encuentran en los grupos focales realizados a los estudiantes con limitación visual, quienes reportan que se encuentran con profesores que los apoyan y que permanentemente buscan nuevas alternativas pedagógicas para favorecer sus aprendizajes ( 7 citas) mientras que otros desconocen cómo enseñarles o se muestran indiferentes frente a sus necesidades de aprendizaje (3 citas). Este tipo de situación en ocasiones se presenta al interior de una misma Institución Educativa. Las siguientes frases dan cuenta de esta situación:

“...mis profesores son pacientes, me guían y me tienen paciencia, a la hora del descanso me explican cosas que no entiendo, me guían, pues 
si, tratan de colaborarle en lo que más pueden, me he sentido bien aquí en el Colegio" y "...por ejemplo, la profesora si tiene que escribir algo en el tablero me dice: "Marcela si tienes quien te dicte?o te ayudo con las lecturas, a copiar o algo asî". como:

Esta práctica realizada por algunos docentes contrasta con otras

\begin{abstract}
“...los profesores no saben cómo explicarle a uno, y uno tiene que quedarse ahí sentado casi toda la tarde esperando a ver a qué hora le explican. "...hay profesores que todavía no, por ejemplo en el aula a ellos se les olvida que hay una persona invidente también ahí y que tienen que explicarle pues para que esa persona también entienda." $y$ sin un profesor es docente se arriesga a tener contacto con uno ino cierto? Deber profesional en todos los sentidos, como la mayoría de los profesores lo son, pero hay un profesor en el colegio, sinceramente que de verdad ni siquiera se arriesga a por lo menos experimentar con uno".
\end{abstract}

Esta última frase evidencia la actitud de indiferencia por parte del profesor que es experimentado por el estudiante con limitación visual.

Los padres de Familia destacan también la existencia de prácticas pedagógicas incluyentes, tales como la realización de adaptaciones curriculares por parte de algunos de los profesores (8 citas), De la misma manera señalan la como una fortaleza de los procesos de inclusión la dedicación (tiempo) que dedican algunos de los profesores para trabajar con el estudiante con limitación visual (1 narrativa). Las siguientes frases ilustran estas afirmaciones:

"le pusieron alquien ahí con él que le explicaba que podía tocar y las cosas que, todo lo que él todo el sabia como se llamaba, le dijeron: esto se llama así, así, entonces es una ventaja y "...nos pone mucha atención (refiriéndose a la maestra de aula), las veces que yo he hablado con ella me ha prestado mucha atención y para poderle comunicar lo de mi hijo porque no le rendía nada académico y yo le dije profesora es que yo me he dado cuenta que él prefiere hacer los manuales y trabajar con las manos que aprender cosas de memoria"

Se encontraron también narrativas que señalan cómo la carencia de preparación de los docentes genera exclusión en el aula, sumado en ocasiones a la falta de compromiso de algunos de ellos. Al respecto los padres se expresaron de la siguiente forma:

“...la profesora la dejaba (se refiere a su hija con limitación visual) que hiciera ahí lo que ella quisiera y tampoco. A ellos también toca exigirles para que aprendan" y"El profesor no entendía el problema que ella tiene, entonces si ha habido inconvenientes con algunos profes".

\section{Significado de la Discapacidad}

Esta categoría da cuenta de la comprensión que han construido los participantes de la condición de discapacidad; las implicaciones que tiene en relación con las dinámicas micro y macro sociales en las que participan.
En el análisis de la narrativa se evidencia la existencia de diferentes significados frente a la condición de limitación visual, a partir de la vivencia de cada participante y de su experiencia frente a ésta condición.

\section{Dominio: una oportunidad difícil de alcanzar}

Es así como para el estudiante con limitación visual, la discapacidad se convierte en una oportunidad para desarrollar diferentes habilidades ( 6 citas), y el espacio escolar es la posibilidad para lograrlo; sin embargo, otras de las narrativas tanto de estudiantes con limitación visual como de sus padres dan cuenta de la dificultad para acceder a una oportunidad en esas instituciones educativas abiertas a las propuestas de inclusión escolar (3 citas). Las siguientes frases de dos estudiantes con limitación visual y de un padre de familia evidencian estas apreciaciones:

"algunas veces he salido al patio con los compañeros...bueno...hay que tomar un riesgo para poder saber si va a pasar algo, si uno no toma el riesgo yo creo que nunca va a llegar a ningún lado, pero si uno no se arriesga no hace nada. Yo me he arriesgado, y pienso que si todos hacemos alguna vez lo mismo...es como para todos... por ejemplo, cuando uno va a presentar una prueba o alguna entrevista para un colegio y yo no me arriesgo para saber que me va a pasar"

"al principio fue duro, porque a los otros niños les daba miedo, habían mamas que decían que no, porque mientras le dedicaban tiempo a ellos, los otros niños estaban perdiendo clases, nosotros sufrimos mucho... a las personas discapacitadas en ninguna institución los atendian".

\section{Dominio: un reto pedagógico.}

Para algunos de los docentes participantes la condición de discapacidad dificulta su quehacer en el aula (8 citas) al respecto uno de ellos se expresó de la siguiente manera:

"No todas las discapacidades son fáciles de integrar, o sea de pronto los invidentes, los sordos, los ciego-sordos eh...sordo-ciegos... ¿es así? hasta los Down... Pero por ejemplo, los que tienen un autismo, déficit cognitivo profundo...como el que tiene eehh, no lo digo porque no hagan nada en el aula".

En este mismo sentido se encuentran narrativas en los docentes que evidencian sentimientos de impotencia cuando se enfrentan al proceso de enseñanza del estudiante con limitación visual, al respecto uno de ellos afirmó:

"Angustia de no saber que material...como trabajarle, que usar, como enseñarle, que metodologías usar. Y fuera de eso como no sé...como ternura, como nostalgia de verlos"

Sin embargo, coexisten otras narrativas que señalan como para otros de los docentes el trabajo con estudiantes con una condición de discapacidad como la limitación visual se, convierte en un reto para su quehacer profesional. La siguiente afirmación ilustra esta apreciación: 
"El reto era mirar a ver si soy capaz de enseñarle a ese niño, sí, a pesar de que no tiene las mismas habilidades que los otros, y descubrir en él que otras habilidades iba a manejar, entonces la verdad siempre ha sido como un reto bien interesante"

\section{DISCUSIÓN}

El Objetivo de esta investigación se centró en comprender las Representaciones Sociales de la comunidad educativa de padres maestros y estudiantes de 10 Colegios del Distrito, en relación con la experiencia de inclusión escolar de niños con limitación Visual, con el propósito de generar, una propuesta educativa orientada a favorecer los procesos de Inclusión en dichos espacios escolares.

El análisis de los resultados en la narrativa obtenida en los grupos focales nos permitió acercarnos a la comprensión de algunos de los contenidos que dan forma a la manera como estos actores sociales comprenden los procesos de inclusión educativa y su forma de actuar en relación con ellos, particularmente en relación con las Necesidades y Expectativas frente al proceso, las Prácticas que ocurren al interior de la escuela, (relacionales y pedagógicas) y por último el significado que han construido frente a la condición de discapacidad.

Los resultados de esta investigación permiten concluir que si bien existen contenidos compartidos en algunas de las representaciones sociales que han construido los diferentes actores de la comunidad educativa en relación con los procesos de inclusión escolar de estudiantes con limitación visual, también evidencian diferencias entre ellos a partir de su rol, experiencia y relación con esta condición.

Es así como existe una representación compartida por todos los actores participantes, frente a las necesidades de capacitación; sin embargo es el grupo de docentes el que demanda una mayor necesidad de capacitación en temáticas específicas tales como el conocimiento de metodologías y estrategias pedagógicas que le permitan reconocer y responder a la diferenciación en el aula, el uso de tecnologías que apoyen los procesos de aprendizaje de sus estudiantes y conocimiento en relación con la discapacidad. Este hallazgo corrobora los resultados reportados en el estudio realizado por La Defensoría del Pueblo (2004), en el que se señala la ausencia de soportes pedagógicos acordes a las necesidades de los estudiantes con discapacidad.

Existe también, particularmente en el grupo de compañeros y de sus padres la necesidad de capacitación dirigida a sensibilizar e informar acerca de la condición de discapacidad visual. Los resultados permiten concluir cómo en la comunidad educativa se encuentran representaciones que dan cuenta de imaginarios, atribuciones y estereotipos negativos alrededor de ésta condición, producto del desconocimiento frente a la misma. Aspecto que explica en gran medida el miedo que sienten los compañeros y algunos de los profesores frente al estudiante con limitación visual y que coincide con los resultados encontrados por Checo y Alto (1995)

Igualmente es importante visibilizar en estos miembros de la comunidad educativa los valores y sentimientos de solidaridad que promueven los procesos de inclusión. Los resultados señalan como algunos de los participantes reconocen en este proceso una posibilidad para construir formas de relación diferentes entre los miembros de una comunidad. Al respecto, Rodríguez y González (2012), han reportado para el caso de personas en condición de discapacidad física, el aporte de la inclusión educativa a la construcción de redes sociales diferentes del entorno meramente familiar, particularmente a partir de las relaciones interpersonales. Sin embargo, llama la atención sobre el hecho de que dichas redes, no son altamente organizadas, pero que abren el camino a mayores niveles de participación.

En cuanto a las necesidades detectadas en relación con los recursos humanos y físicos que permiten hacer realidad los procesos de inclusión escolar, se concluye que todos los participantes, pero especialmente los docentes y los padres valoran el trabajo realizado tanto por los profesores de apoyo como por el INCI, que como Institución representativa del Estado tiene la función de diseñar políticas destinadas al mejoramiento de la calidad de la población con limitación visual; sin embargo las necesidades que se presentan en la cotidianidad de las Instituciones Educativas desbordan los recursos de los que disponen, de tal manera que el apoyo Institucional resulta insuficiente. Es así, como sumado a los sentimientos de soledad expresados por los docentes, producto del desconocimiento de cómo hacer realidad la inclusión en el aula, existe en ellos una representación de sobrecarga laboral, que se traduce en formas de relación excluyentes en su práctica pedagógica. Este mismo sentimiento de soledad y sobrecarga laboral es expresado también por los profesores de apoyo. Situación que permite concluir, que es fundamental generar formas diferentes de trabajo al interior de las instituciones escolares, en las que se privilegie el trabajo en grupo; es posible pensar que a través del fortalecimiento de equipos interdisciplinarios facilitadores de los procesos de inclusión educativa, cuya función se centre en fortalecer al profesor de aula, a través de la discusión de metodologías y estrategias pedagógicas que favorezcan la participación y el aprendizaje de sus estudiantes se construirá una escuela incluyente. Finalmente, el profesor de aula es el actor principal de estos procesos de inclusión. Como se señaló anteriormente el trabajo que realiza la profesora de apoyo es valorado positivamente por la comunidad educativa, pero está más orientado, en la actualidad, a dar respuesta a las necesidades particulares del estudiante con limitación visual, que sin duda alguna son importantes, más que al fortalecimiento del profesor de aula.

Se encontraron también diferentes representaciones en relación con la necesidad de realizar adaptaciones en el espacio físico de la institución educativa, mientras que para los profesores éstas no cuentan con instalaciones adecuadas, los estudiantes con limitación visual, en su mayoría, opinan que las instalaciones son adecuadas. El colegio es un espacio más dentro de una ciudad como Bogotá, (que en este aspecto es poco incluyente), en la cual deben movilizarse los estudiantes con limitación visual, probablemente esto hace difícil para ellos percibir esta necesidad. Sin duda es importante considerar un diseño arquitectónico incluyente como parte de la política pública que se dirige a regular los procesos de inclusión educativa.

El reconocimiento de que los procesos de inclusión involucran a la comunidad educativa en su conjunto, es decir que van más allá de 
7 la práctica puramente pedagógica, de la relación estudiante-docente, hace pensar en la necesidad de reflexionar sobre las prácticas que ocurren al interior de las instituciones. La inclusión es un espacio de interacción, y en ese sentido permite pensar en nuevas formas de relación y de construcción de valores sociales como la solidaridad y el respeto a la diferencia que, como lo señalan los resultados se genera en muchos de los compañeros, pero coexisten también con prácticas relacionales excluyentes que ocurren en la cotidianidad del espacio educativo, especialmente entre los compañeros. Es importante intervenir a este nivel, pues, muchas de las tensiones detectadas en el trato diario se originan en el desconocimiento, que se traduce en la presencia sentimientos de miedo y rechazo frente a las interacciones con los estudiantes con limitación visual; los cuales pueden ser resignificados, no solamente a partir de la información sobre esta condición, sino de la utilización de una propuesta pedagógica diferente. En ese sentido es posible plantear que metodologías de aprendizaje cooperativo que valoren la interdependencia en las interacciones de los estudiantes, se conviertan en una posibilidad interesante que debe ser considerada como práctica institucional que seguramente, incidirá en el tipo de relaciones que se establecen entre los compañeros, así como entre éstos y los docentes.

La cotidianidad del contexto escolar debe hacer visible, la participación de todos sus miembros, sin duda este tipo de propuesta a largo plazo generará trasformaciones culturales que valoren realmente la diferencia. La utilización de estrategias de comunicación que se orienten a favorecer prácticas relacionales incluyentes a través de campañas promovidas desde la misma Secretaria de Educa- ción contribuirá, muy seguramente, a la construcción de esta nueva cultura.

En relación con las prácticas pedagógicas, se detecta en los docentes una tensión expresada en representaciones que dan cuenta del compromiso frente al estudiante con limitación visual en contraste con otras representaciones que expresan la frustración e impotencia que sienten frente a ellos, y que se traduce en prácticas pedagógicas excluyentes, como se muestra en los resultados. Los docentes señalan que no existe coherencia en la política de inclusión pues finalmente no sienten apoyo institucional. Los estudiantes con limitación visual, se convierten en su responsabilidad y ellos no cuentan con las herramientas ni el tiempo para responder a sus necesidades. Una propuesta de capacitación dirigida a los docentes, que se oriente a proporcionar herramientas pedagógicas concretas para ser utilizadas en el aula, permitirá pensar en la transformación de estas prácticas.

Para los estudiantes y padres, la Inclusión supone un derecho que debe ser exigido, es decir que no existe en la sociedad un reconocimiento claro del mismo, situación a la que se enfrentan por ejemplo cuando solicitan un cupo para sus hijos en un plantel educativo; lo cual señala la necesidad de fortalecer la socialización e interiorización de la política de inclusión en los diferentes sectores de la sociedad. Existe también coincidencia en el contenido de la representación en relación con el reconocimiento de la inclusión educativa como un camino para la transformación cultural. . 


\section{Referencia:}

Las referencias a otras obras son una parte Muy importante en la literatura científica;
ya que estas permiten conocer más sobre los autores y
mantener vivas sus voces dentro del texto.

1. Ashworth, P (2008).Conceptual Foundations of Qualitative Psychology.En J, Smith (Ed). Qualitative Psychology (pp44-25). Los Angeles: SAGE.

2. Blanco, R. Inclusión educativa en America Latina y el Caribe. CEE Participacion Educativa, 18, pp. 46-59

3. Buitrago. J (2011).Plan estratégico 2011-2014. "Inclusión educativa y sociocultural de la población con discapacidad visual”. Bogotá: INCI.

4. Departamento Nacional de Planeación Documento. COMPES Social: Política Pública Nacional de Discapacidad. Recuperado el 6 de Noviembre de 2012 de http://www..areandina.edu.co

5. Bogad, R. \& Taylor, S. (1994). Introducción a los Métodos Cualitativos de Investigación. Barcelona: Paidos

6. Checa, F. \& Ato, M. (1995). Relaciones socioafectivas de alumnos ciegos y con baja visión en aulas ordinarias. Estudio sociométrico. Revista Integración, 18, 25-36.

7. Defensoría del Pueblo (2004).La integración educativa de niños y niñas con discapacidad. Una evaluación en Bogotá desde la perspectiva de derechos. Bogotá: Imprenta Nacional de Colombia.

8. Delgado, M. (2007).Prácticas y representaciones sociales acerca de la ceguera en la ciudad de Cali. Manuscrito no publicado. Universidad del Valle: Cali.

9. García, C. \& García, E. (1991).Situación de alumnos con déficits visuales en aulas integradas. Revista Integración, 5. 38-47

10. Jodelet, D. (1987). La Representación Social, Conceptos, Fenómenos y Teorías. En S. Moscovici. Psicología social (Vol. 2 pp.135-146). Buenos Aires: Paidós.

11. Ministerio de Educación Nacional. (1994). Ley General de Educación Santafé de Bogotá.

12. Moreno, M. (2007). Inclusión Social de las .Personas con discapacidad: Reflexiones, Realidades, Retos. Bogotá: Universidad Nacional de Colombia.

13. Moscovici, S. (1987). Psicología social II. Buenos Aires: Paidós.

14. Quinn,G. \&, Degener,T. (2002). Uso actual y Posibilidades Futuras de los Instrumentos de Derechos Humanos de las Naciones Unidas en el Contexto de Discapacidad. Recuperado el día 11 de Abril de 2009 en la págin http://www.unhchr.ch/html/menu6/2/disability_sp.pdf
15. Rodriguez, D \& Gonzalez, M. (2012). Exploración de la experiencia de empoderamiento en personas con discapacidad física integradas al aula regular. Revista Psicogente, 15, (27), pp. 153 - 157.

16. Secretaría Distrital de Educación. (1997). Educación para todos. Lineamientos generales para la atención educativa a la población con limitaciones o capacidades excepcionales en los centros educativos del distrito. Secretaría de Educación de Santafé de Bogotá Distrito Capital.

17. Shea, T. \& Bauer, A. (1999). Educación Especial. Un enfoque ecológico. $\left(2^{\mathrm{a}}\right.$ Ed.). México: McGrawHill.

18. Spradley, J (1979). The Ethnographic Interview. Holt: Rinehart and Winston Inc

19. Unesco. (1990). Declaración Mundial sobre la Educación Para Todos y Marco de Acción Para Satisfacer las Necesidades Básicas de Aprendizaje. Nueva York.

20. Vaca, P,,Chaparro,, V \&Pérez, N. (2006). Representaciones Sociales acerca de la Identidad de Género de una mujer que emplea la Violencia en la Solución de Conflictos. Psicología desde el Caribe, 18 23-57

21. Vaca, P., Córdoba, L., Rosero, R., Gómez-Benito, J., Escobar, N. \&Lucas, R (2011). Creencias y prácticas de Mujeres con discapacidad frente al ejercicio de sus derechos. Estudios de Psicología, 32(2) 209-226

22. Vallejo, I. (2005). Parecerse a nosotros. Debates acerca de las representaciones sociales de docentes de educación básica acerca de la discapacidad. En P. Vainy \& A. Rosato, Comunidad, discapacidad y exclusión social. La construcción social de la normalidad. Alteridades, diferencias y diversidad. (pp.87-93). Buenos Aires: Ediciones Novedades Educativas.

23. Valles, M (1997). Técnicas cualitativas de Investigación Social. Madrid: Síntesis.

24. Verdugo, M. A. (2003). La concepción de discapacidad en los modelos sociales. En M. A. Verdugo \& F. B. Jordán deUrríes Vega (Coords.), Investigación, innovación y cambio. $V$ Jornadas Científicas sobre personas con discapacidad (pp. 235- 247). Salamanca: Amarú.

25. Wilkinson, S. (2008). Focus Groups.En J, Smith (Ed). Qualitative Psychology (pp 186-206). Los Angeles: SAGE

26. Woolfok, A. (1999). Psicología Educativa. ( $7^{\mathrm{a}}$ Ed.). México: Prentice Hall.

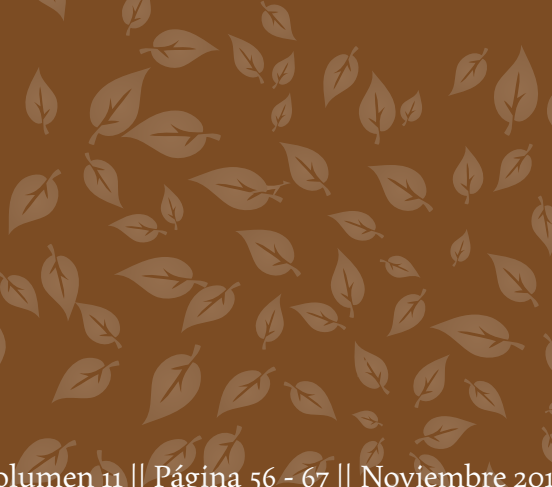
Sexico:

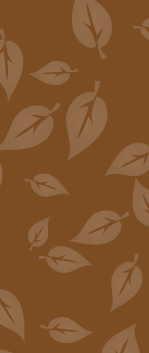

